\title{
Detecting Heavy Charged Higgs boson at the LHC
}

\author{
Monoranjan Guchait*, Aravind H Vijay \\ Tata Institute of Fundamental Research \\ Homi Bhabha Road, Mumbai 400005, India. \\ E-mail: guchaitetifr.res.in
}

\begin{abstract}
Signature of heavier charged Higgs boson, much above the top quark mass, is investigated at the LHC Run 2 experiments, following its decay mode via top and bottom quark focusing on both hadronic and semi-leptonic signal final states in the context of generic two Higgs doublet model with a special emphasis on supersymmetry motivated Type II model. The signal is found to be heavily affected by huge irreducible standard model backgrounds due to the top quark pair production and QCD events. The jet substructure technique is used to tag moderately boosted top jets in order to reconstruct charged Higgs mass. The simple cut based analysis shows very poor sensitivity. However, employing the multi-variate analysis(MVA) technique, a remarkable improvement in signal sensitivity is achieved. We find that the charged Higgs signal for the mass range about $300-600 \mathrm{GeV}$ is observable with $1000 \mathrm{fb}^{-1}$ luminosity. However, for high luminosity, $\mathscr{L}=3000 \mathrm{fb}^{-1}$, the discovery potential can be extended to $700-800 \mathrm{GeV}$.
\end{abstract}

The 39th International Conference on High Energy Physics (ICHEP2018)

4-11 July, 2018

Seoul, Korea

${ }^{*}$ Speaker. 


\section{Introduction}

Among the plethora of BSM candidates, the supersymmetry based models, such as the minimal supersymmetric standard model(MSSM) is the most popular and very well studied BSM scenario. The MSSM requires at least two Higgs doublets to make the theory anomaly free, and also to generate the masses of up and down type of fermions. In general, two Higgs doublet model(2HDM) consisting of an extra SU(2) Higgs doublet added with the SM Higgs doublet, is well motivated and consistent with the Higgs discovery. Generally, 2HDM is classified into four categories, Type I, II, III and IV depending on the nature of Yukawa couplings, subject to $Z_{2}$ symmetry in order to avoid Flavor changing neutral current. In all classes of 2HDM scenario, there exist five physical Higgs boson states, two CP even ( $h, H$, with the assumption, $\left.m_{h}<m_{H}\right)$, one CP odd $(A)$, and two charged Higgs bosons $\left(H^{ \pm}\right)$, where the lightest $\mathrm{CP}$ even Higgs $h$ can be interpreted as the SM-like Higgs boson in the decoupling limit, where the other states can be very heavy, much above the electroweak scale $[1,3]$. Looking for the charged Higgs boson signal is unique, since its discovery clearly, and unambiguously confirms the presence of BSM. In this presentation, we report the detection prospect of charged Higgs boson for the intermediate to heavier mass range, 300-1000 $\mathrm{GeV}$, considering the decay mode, $H^{+} \rightarrow t \bar{b}$ with the hadronic and leptonic final state. Since the top quark from $H^{ \pm}$decay is boosted, the jet substructures techniques are employed to tag top jets, and subsequently reconstructed the charged Higgs boson. In the study [2], we found that MVA based analysis predicts an improved sensitivity. Finally results are presented for general 2HDM for categories.

\section{Signal and Bacnkground}

In the intermediate to heavier mass range $\left(m_{H^{ \pm}} \gtrsim m_{t}\right)$, the charged Higgs is produced directly in proton-proton collision via the process,

$$
p p \rightarrow t H^{-}+X
$$

At the parton level, the production mechanism is initiated via two subprocesses,

$$
\begin{aligned}
& g g, q \bar{q} \rightarrow t \bar{b} H^{-}(4 \mathrm{FS}) \\
& g b \rightarrow t H^{-} \quad(5 \mathrm{FS})
\end{aligned}
$$

in 4 flavor(4FS) and 5 flavor scheme(5FS) at the leading order(LO) respectively. At finite order, the cross section in 4FS does not match with 5FS, as expected, due to different ways of treating perturbative calculation. The prescription to match these cross sections computed in two schemes is given by $[?, ?]$,

$$
\sigma=\frac{\sigma^{4 \mathrm{FS}}+w \sigma^{5 \mathrm{FS}}}{1+w} \text { with } w=\ln \frac{m_{H^{ \pm}}}{m_{b}}-2
$$

Similarly, the theoretical uncertainties are combined With this matching methodology, the overall theoretical uncertainty of the combined NLO cross section is found to be around $10 \%$, where as 
Figure 1: MVA output (D) distribution for signal and backgrounds corresponding to hadronic signal final state and $m_{H^{ \pm}}=500 \mathrm{GeV}, \tan \beta=30$ for Type II $2 \mathrm{HDM}$.
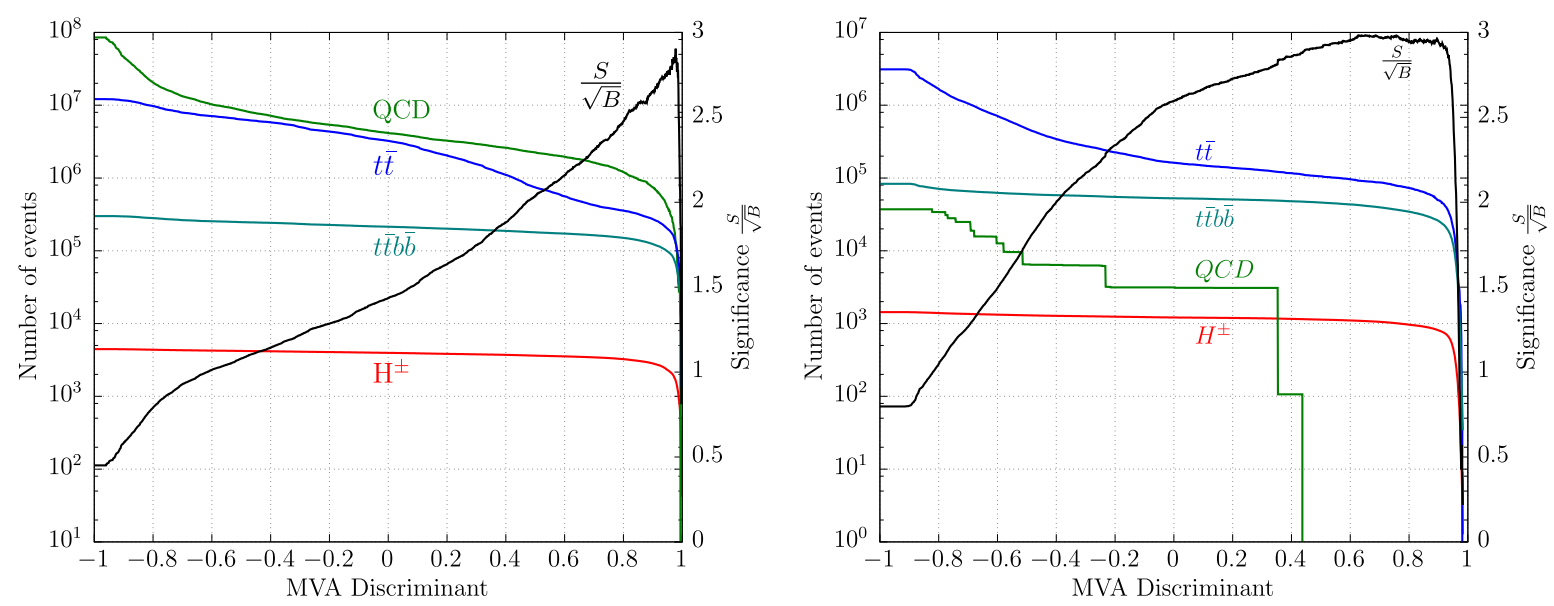

Table 1: Significances for hadronic (leptonic) final states and for three luminosity options, in SUSY motivated Type II model with $\tan \beta=30$.

\begin{tabular}{|l|cccc|}
\hline & \multicolumn{4}{|c|}{$S / \sqrt{B}$} \\
$m_{H^{ \pm}}(\mathrm{GeV}) \rightarrow$ & 300 & 500 & 800 & 1000 \\
\hline $\mathscr{L}=300 \mathrm{fb}^{-1}$ & $6.1(5.2)$ & $2.7(2.94)$ & $0.61(0.96)$ & $0.22(0.39)$ \\
$\mathscr{L}=1000 \mathrm{fb}^{-1}$ & $11.0(9.5)$ & $4.8(5.4)$ & $1.1(1.7)$ & $0.40(0.71)$ \\
$\mathscr{L}=3000 \mathrm{fb}^{-1}$ & $19.1(16.5)$ & $8.4(9.3)$ & $1.9(3.0)$ & $0.70(1.2)$ \\
\hline
\end{tabular}

the individual 4FS and 5FS cross sections at NLO are in reasonable agreement within $\sim 20 \%$ from the central value [4]. We focus the charged Higgs signal final state in two categories:

$$
\begin{aligned}
& \mathrm{a}: H_{\mathrm{reco}}^{ \pm}+t_{\text {reco }}+\mathrm{n}_{\mathrm{b}}(\geq 1) \mathrm{b}-\text { jet } \\
& \mathrm{b}: H_{\text {reco }}^{ \pm}+\mathrm{n}_{\ell}(\geq 1)+\mathrm{n}_{\mathrm{b}}(\geq 1) \mathrm{b}-\text { jet }
\end{aligned}
$$

where $H_{\text {reco }}^{ \pm}$and $t_{\text {reco }}$ represent the reconstructed Charged Higgs and top quark, and $n_{\ell}$ and $n_{b}$ are the number of leptons and $b$-jets respectively, and required to be at least one. The main dominant source of irreducible SM backgrounds are due to $t \bar{t}$, and inclusive hard QCD jet production. The QCD jet production becomes dominant source of irreducible background, in particular corresponding to the hadronic signal final state, due to the non-negligible mis-tagging probability of hard jets as a top jet. Details of event generation and selections are discussed in Ref. [2]. We use MVA technique to improve the signa;1 sensitivity. In Fig. 1, we present the distribution of MVA discrimiantor. The selection of $D>0.9$ leads to a significance $\sim 2.65 \sigma$ which goes up more for higher luminosity options for hadronic case, where as for leptonic case, it becomes about $3 \sigma$ for $\mathscr{L}=300 \mathrm{fb}^{-1}$. The study is extended upto the $1000 \mathrm{GeV}$ mass of the charged Higgs. Signal significances are presented for both hadronic and leptonic final state(in parenthesis) in Table.1 for three masses of charged Higgs and for three integrated luminosity options. The Table 1 shows that the signature of 
charged Higgs of mass around $800 \mathrm{GeV}$ is observable in leptonic channel for $3000 \mathrm{fb}^{-1}$ luminosity option unlike the hadronic final state. For lower range of masses $(\sim 500 \mathrm{GeV})$ signal is feasible even for $1000 \mathrm{fb}^{-1}$ luminosity option. Scaling the cross sections for classes of $2 \mathrm{HDM}$, the signal significances are presented for all types of models. The discoverable region in the $\tan \beta-m_{H^{ \pm}}$ plane are presented in Ref [2].

\section{Summary}

In this study, we explore the detection prospect of the charged Higgs boson for the heavier mass range at the LHC with the center of mass energy, $\sqrt{s}=13 \mathrm{TeV}$, within the framework of generic 2HDM. The signature of charged Higgs is analyzed for the final state consisting of a reconstructed charged Higgs mass and extra $b$-jets plus an additional reconstructed top quark for hadronic events, while in leptonic events, a lepton is required without reconstruction of the second top. Remarkably, MVA analysis yields substantial improvement in signal significance. For example, this MVA based analysis shows that with $\mathscr{L}=1000 \mathrm{fb}^{-1}$, the signature of charged Higgs boson for the mass range $\sim 300-700 \mathrm{GeV}$ can be probed for both hadronic and leptonic channel. For more higher luminosity option, such as $3000 \mathrm{fb}^{-1}$, the discovery reach of $m_{H^{ \pm}}$can be extended up to $\sim 800 \mathrm{GeV}$ for hadronic final state, where as for leptonic case, it can be extended further, up to almost $1 \mathrm{TeV}$ for high values of $\tan \beta$. The discovery potential of charged Higgs boson are presented in the $m_{H^{ \pm}}-\tan \beta$ plane for a few integrated luminosity options in Ref [2]. This figure indicates that the discovery reach corresponding to leptonic final state is better than the hadronic signal case. The results show that for high $\tan \beta=30$ scenario, it is difficult to achieve any detectable signal sensitivity, except for Type II and Type IV models. Indeed, it is hard to discover the signal of the charged Higgs boson of mass beyond $800 \mathrm{GeV}$ for low $\tan \beta$ scenario, even for higher luminposity options.

\section{References}

[1] J. F. Gunion, S. Dawson, H. E. Haber, and G. L. Kane, “The Higgs hunter's guide”, vol. 80. Brookhaven Nat. Lab., Upton, NY, 1989.

[2] Monoranjan Guchait, Aravind H. Vijay,"Probing Heavy Charged Higgs Boson at the LHC", arXiv:1806.01317 [hep-ph].

[3] G. C. Branco, P. M. Ferreira, L. Lavoura, M. N. Rebelo, M. Sher, and J. P. Silva, "Theory and phenomenology of two-Higgs-doublet models”, Phys. Rept. 516 (2012) 1-102, arXiv:1106.0034 [hep-ph].

[4] M. Flechl, R. Klees, M. Kramer, M. Spira, and M. Ubiali, "Improved cross-section predictions for heavy charged Higgs boson production at the LHC," Phys. Rev. D91 no. 7, (2015) 075015, arXiv:1409.5615 [hep-ph].

[5] T. Sjostrand, S. Mrenna, and P. Z. Skands, "PYTHIA 6.4 Physics and Manual," JHEP 05 (2006) 026, arXiv:hep-ph/0603175 [hep-ph]

[6] J. Alwall, R. Frederix, S. Frixione, V. Hirschi, F. Maltoni, O. Mattelaer, H. S.Shao, T. Stelzer, P. Torrielli, and M. Zaro, "The automated computation of tree-level and next-to-leading order differential cross sections, and their matching to parton shower simulations," JHEP 07 (2014) 079, arXiv:1405.0301 [hep-ph]. 